\title{
Scale for the Phase Diagram of Quantum Chromodynamics
}

\author{
Sourendu Gupta, ${ }^{1}$ Xiaofeng Luo, ${ }^{2,3}$ Bedangadas Mohanty, ${ }^{4}$ \\ Hans Georg Ritter, ${ }^{3} \mathrm{Nu} \mathrm{Xu}^{5,3}$ \\ ${ }^{1}$ Department of Theoretical Physics, Tata Institute of Fundamental Research, \\ Homi Bhabha Road, Mumbai 400005, India \\ ${ }^{2}$ Department of Modern Physics, University of Science and Technology of China, \\ Hefei 230026, China \\ ${ }^{3}$ Nuclear Science Division, Lawrence Berkeley National Laboratory, Berkeley, CA 94720, USA \\ ${ }^{4}$ Experimental High Energy Physics and Applications Group, Variable Energy Cyclotron Centre, \\ 1/AF Bidhan Nagar, Kolkata 700064, India \\ ${ }^{5}$ College of Physical Science and Technology, Central China Normal University, \\ Wuhan 430079, China
}

Matter described by Quantum Chromodynamics (QCD), the theory of strong interactions, may undergo phase transitions when its temperature and the chemical potentials are varied. QCD at finite temperature is studied in the laboratory by colliding heavy-ions at varying beam energies. We present a test of QCD in the non-perturbative domain through a comparison of thermodynamic fluctuations predicted in lattice computations with the experimental data of baryon number distributions in high-energy heavy-ion collisions. This study provides evidence for thermalization in these collisions, and allows us to find the crossover temperature between normal nuclear matter and a deconfined phase called the quark gluon plasma. This value allows us to set a scale 


\section{for the phase diagram of QCD.}

QCD is the theory of strong interactions - one of the four fundamental interactions occurring in nature, and an essential part of the standard model of particle physics. It describes interactions between quarks and gluons, which are the ultimate constituents of the majority of the visible mass of the universe $(1,2)$. In the short-distance regime where the momentum exchange between quarks and gluons is large, the strong coupling constant becomes small through the mechanism of asymptotic freedom. In this perturbative region QCD is very successful in explaining various processes observed in experiments involving electron-positron, proton-proton and proton-antiproton collisions (3). In the non-perturbative regime tests of the theory were related to the computation of hadron properties (4). In other regimes of long-distance nonperturbative physics, the theory is yet to be tested. Here, we test the thermodynamics of bulk strongly interacting matter.

Experimental tests of non-perturbative QCD in the bulk can be carried out by colliding heavy-ions (like $\mathrm{U}, \mathrm{Pb}, \mathrm{Au}$, and $\mathrm{Cu}$ ) at different center of mass energies, $\sqrt{s_{N N}}(5-8)$. Several experimental programs have been launched or are in the planning stage at facilities such as the Large Hadron Collider (LHC), the Relativistic Heavy Ion Collider (RHIC), the Super Proton Synchrotron (SPS), the Facility for Anti-proton and Ion Research (FAIR) and the Nuclotronbased Ion Collider fAcility (NICA), where the essential features of the QCD phase diagram can be studied.

In QCD there are conserved quantities like the net-baryon number, $B$, the net-electric charge, $Q$, and the net-strangeness, $S$. The term net means the algebraic sum of the quantum numbers, where those of anti-particles are the negatives of the corresponding particles. As a result the thermodynamics of the bulk can be characterized by the corresponding chemical potentials (energy needed to add/remove one unit of the conserved quantity to/from the system) $\mu_{B}, \mu_{Q}$, and $\mu_{S}$ in addition to the temperature, $T$, conjugate to the conserved energy of a bulk 
system. In experimental studies of particle ratios measured in heavy-ion collisions it is observed that the relevant values of $\mu_{Q}$ and $\mu_{S}$ are small compared to $\mu_{B}$. For example, in Au ion collisions within rapidity range of \pm 0.1 unit at $\sqrt{s_{N N}}=200 \mathrm{GeV}$ (with impact parameter less than $3 \mathrm{fm}$ ) one finds that $\mu_{B}=22 \pm 4.5 \mathrm{MeV}$, while $\mu_{S}=3.9 \pm 2.6 \mathrm{MeV}$ and $\mu_{Q}$ is still smaller (9).

The lattice formulation of QCD is a non-perturbative approach from first principles for obtaining the predictions of QCD. Space-time is replaced by a lattice; quarks occupy the sites, and gluons occupy the links between the sites. The lattice spacing, $a$, is the inverse of the cutoff required to regulate any interacting quantum field theory. The theory is solved numerically at several values of $a$. The extrapolation to the continuum $(a=0)$ can then be made through the renormalization group equations. In QCD there is a conventional temperature, $T_{c}$, which is an intrinsic scale of bulk hadronic matter. We follow the definition that it is the temperature at the peak of a susceptibility related to the confinement-deconfinement order parameter (called the Polyakov loop susceptibility, $\left.\chi_{L}\right)$ at $\mu_{B}=0$ (10-13). Lattice QCD computations show that this peak is finite, which corresponds to a cross-over $(14,15)$. The temperature at which $\chi_{L}$ peaks, of course, changes with $\mu_{B}$. However, once $T_{c}$ is known, such shifts as a function of $\mu_{B}$ can be quantified. This is similar to saying that the Celsius scale of temperature is defined by the boiling point of water at normal pressure, $P$, and that the boiling point changes with $P$.

One of the most basic questions to ask about bulk hadronic matter is the value of $T_{c}$. This can be represented as a link in a "circle of reasoning" that encompasses all the regimes of non-perturbative QCD (Fig.1). So far the strategy to find $T_{c}$ has been indirect: first lattice QCD computations are performed at both $T=0$ and $T>0$ in order to determine a ratio $T_{c} / m$, where $m$ is a typical hadronic scale (step (b) of Fig.1). Then one replaces the scale $m$, determined on the lattice, by an experimental measurement (step (a)). The temperature at each $\sqrt{s_{N N}}$ extracted from models of particle yields $(18,19)$ is step $(\mathrm{d})$ of the circle of reasoning. From such models one finds that the fireball of bulk nuclear matter created in heavy-ion collisions, which is initially 


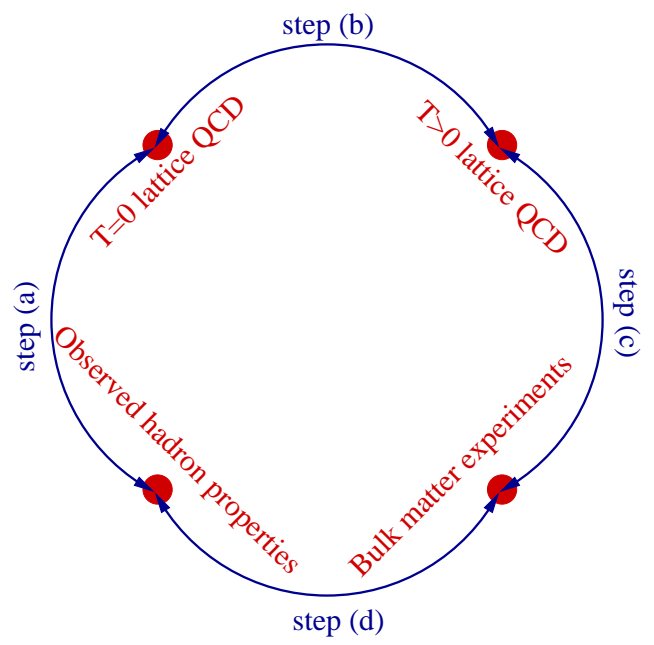

Figure 1: Illustration of the chain of reasoning for testing QCD in the non-perturbative domains of the strong interactions and obtaining the scale, $T_{c}$, of the QCD phase diagram.

out of equilibrium, evolves to a state of thermal equilibrium at chemical freeze-out. The models do no give $T_{c}$; however, they allow the extraction of $T$ and $\mu_{B}$ at freezeout. In this paper we show that predictions of lattice computations of finite temperature QCD (16), taken in conjunction with determinations of $T_{c}$ in step (b) (10-13) agree well with experimental measurements on bulk hadronic matter (17). This allows us to invert the reasoning and extract $T_{c}$ directly from the experimental measurements in heavy-ion collisions (step (c)). The agreement of the temperature from steps (c) and (d) along with the agreement of $T_{c}$ extracted from steps (a) and (b) with that from (c) show the complete compatibility of a single theory of hadron properties and of bulk QCD matter, i.e., of all non-perturbative regimes of the strong interactions. This approach may present a new domain of tests of the standard model of particle physics.

The conjectured phase diagram of QCD: In the current conjectures for the parts of the phase diagram that is accessible with heavy-ion collisions (20) (Fig.2), calculations within simplified models which mimic QCD show that at large $\mu_{B}$ there is a first order hadron-QGP phase transition. This phase boundary is expected to end in a critical point at finite $\mu_{B}$, as lattice com- 


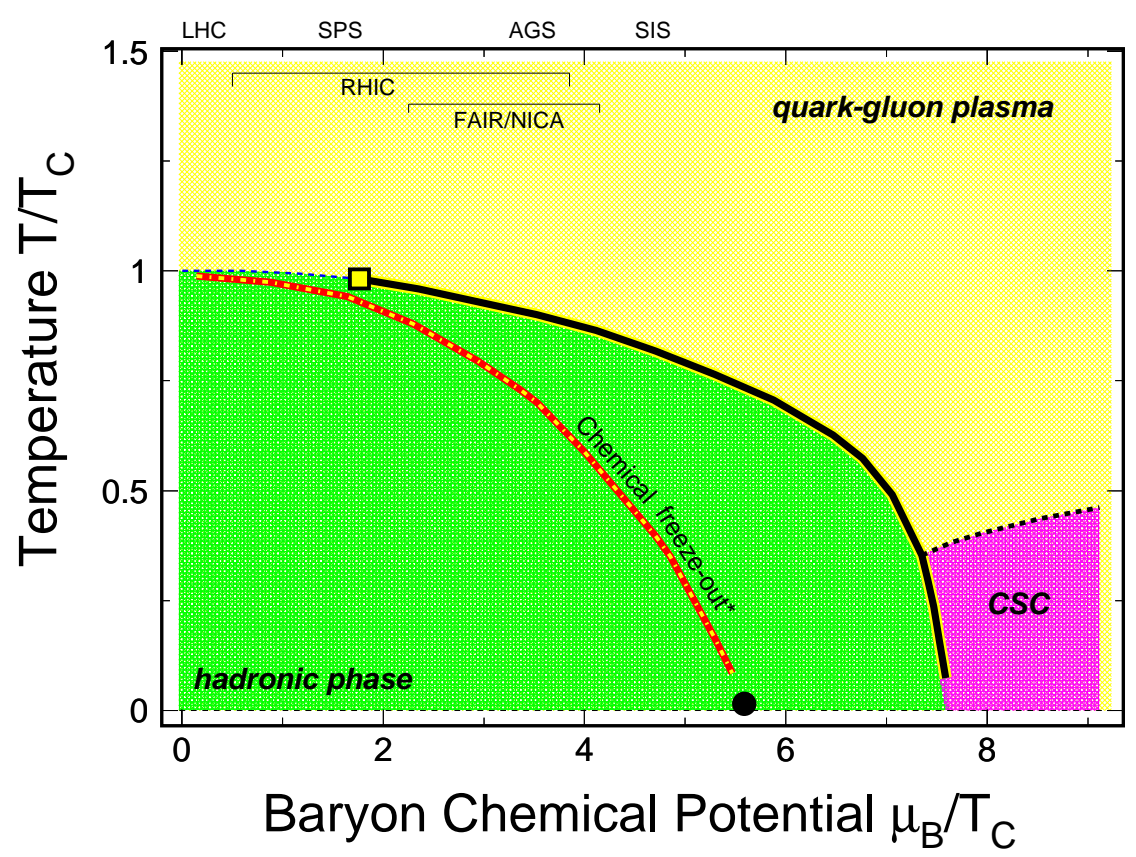

Figure 2: Current conjectures for the QCD phase diagram. The phase boundary (solid line) between the normal low-temperature hadronic phase of bulk QCD matter and the high-temperature partonic phase is a line of first order phase transitions which begins at large $\mu_{B}$ and small $T$ and curves towards smaller $\mu_{B}$ and larger $T$. This line ends at the QCD critical point whose probable position, derived from lattice computations, is marked by a square. At even smaller $\mu_{B}$ there are no phase transitions, only a line of cross-overs (shown by a dashed line). The red-yellow dotted line corresponds to the chemical freeze-out line from the evolution of the bulk QCD matter produced in high energy heavy-ion collisions. The solid point at $T=0$ and $\mu_{B}=938 \mathrm{MeV}$ represents nuclear matter in the ground state. At large $\mu_{B}$ and low $T$ is the color superconductor phase (CSC) (26). 
putations (10-13) agree with general symmetry arguments $(21)$, which indicate that at $\mu_{B}=0$ there is neither a first-order nor a second-order phase transition but only a cross-over at $T_{c}$. The determination of $T_{c}$ sets the scale of the QCD phase diagram. Current best estimates of the position of the critical point (22) are reflected in the position indicated in Fig.2. The experimental focus currently is on an attempt to locate the critical point and the line of phase coexistence $(23,24)$.

By changing $\sqrt{s_{N N}}$ one traces out a line of chemical freeze-out in the phase diagram, as shown in Fig.2. This line is parameterized through a hadron resonance gas model $(18,19)$. Because this work focuses on making a connection between QCD thermodynamic calculations and observables measured in experimental facilities, we also show in Fig.2 the range of $\mu_{B}$ values covered by various experiments as one traverses the chemical freeze-out line by changing $\sqrt{s_{N N}}$. The solid point around $\mu_{B}=938 \mathrm{MeV}$ is the location of ordinary nuclear matter (25), the best characterized point on the phase diagram.

Comparison of experimental measurements with lattice QCD predictions: Lattice QCD computations leave open the question of a scale and yield dimensionless predictions, for example for $P / T^{4}$ as a function of $T / T_{c}$ and $\mu_{B} / T$. Here we discuss the non-linear susceptibilities (NLS) of baryons, $\chi_{B}^{(n)}$, of order $n(27)$. These are the Taylor coefficients in the expansion of $P$ with respect to $\mu_{B}$ at fixed $T$ in the usual dimensionless form

$$
T^{n-4} \chi_{B}^{(n)}\left(\frac{T}{T_{c}}, \frac{\mu_{B}}{T}\right)=\left.\frac{1}{T^{4}} \frac{\partial^{n}}{\partial\left(\mu_{B} / T\right)^{n}} P\left(\frac{T}{T_{c}}, \frac{\mu_{B}}{T}\right)\right|_{T / T_{c}}
$$

Lattice measurements of the series expansion of the NLS in powers of $\mu_{B} / T$ are resummed using Pade approximants in order to give predictions for the above quantities (16). They are of interest because they are related to cumulants of the fluctuations of the baryon number in thermal and chemical equilibrium in a grand canonical ensemble. 
The $n$-th cumulant of such fluctuations, $\left[B^{n}\right]$, is given by

$$
\left[B^{n}\right]=V T^{3} T^{n-4} \chi_{B}^{(n)}\left(\frac{T}{T_{c}}, \frac{\mu_{B}}{T}\right)
$$

where $V$ is the volume of the observed part of the fireball. Because observed hadrons are in thermal and chemical equilibrium at the freeze-out, this relation should hold for cumulants of the observed event-by-event distribution of net-baryon number in heavy-ion collisions. The cumulants are often reported as the variance, $\sigma^{2}=\left[B^{2}\right]$, the skewness, $S=\left[B^{3}\right] /\left[B^{2}\right]^{3 / 2}$ and the Kurtosis, $\kappa=\left[B^{4}\right] /\left[B^{2}\right]^{2}$. It is clear from these definitions that the $V$-dependence in Eq. 2 gives the correct volume scaling expected from the central limit theorem. This leads to the classic extraction of the susceptibility from fluctuations in the grand canonical ensemble $(28,29)$.

There is one remaining subtlety in comparing lattice computations with experimental data. Most experiments are designed to measure event-by-event net-protons. The data discussed in the current work is from the STAR experiment at RHIC (17), which identifies protons and antiprotons by measuring the specific ionization energy loss of these particles in the gas of a Time Projection Chamber. These measurements miss neutrons, the other dominant part of the baryon distribution. This may impose limitations on our measurement of fluctuations. However the effect of isospin fluctuations on the shape of the net-baryon distributions is small (30). Hence we proceed under the assumption that the shape of the net-proton distributions reflects the netbaryon distributions up to distortions smaller than the estimated errors in measurements of the cumulants.

We are unable to exploit Eq. 2 directly in heavy-ion experiments because the volume, $V$, is hard to determine precisely experimentally. However, the ratios

$$
\begin{aligned}
& \left(m_{1}\right): \quad S \sigma=\frac{\left[B^{3}\right]}{\left[B^{2}\right]}=\frac{T \chi_{B}^{(3)}}{\chi_{B}^{(2)}}, \\
& \left(m_{2}\right): \quad \kappa \sigma^{2}=\frac{\left[B^{4}\right]}{\left[B^{2}\right]}=\frac{T^{2} \chi_{B}^{(4)}}{\chi_{B}^{(2)}}
\end{aligned}
$$




$$
\left(m_{3}\right): \quad \frac{\kappa \sigma}{S}=\frac{\left[B^{4}\right]}{\left[B^{3}\right]}=\frac{T \chi_{B}^{(4)}}{\chi_{B}^{(3)}}
$$

do not contain the volume and therefore provide a direct and convenient comparison of experiment and theory (31). The above equations are written in a form that emphasizes this connection - the left hand side can be measured in an experiment whereas the right hand side can be predicted by lattice QCD. We use the notation $m_{1,2,3}$ generically to refer to either side.

We now proceed to discuss the comparison of $m_{1}$ and $m_{2}$ from experiment and theory (Fig.3). The experimental measurements (17) were made using the number of protons $(p)$ and anti-protons $(\bar{p})$ produced in the collision of Au ions around $90^{\circ}$ to the beam axis with the impact parameter of the collisions being less than $3 \mathrm{fm}(32) . p$ and $\bar{p}$ are in the range of $400 \mathrm{MeV} / c$ to $800 \mathrm{MeV} / c$. This choice of momentum range is designed to obtain the purest sample of $p$ and $\bar{p}$. A large fraction of $p$ and $\bar{p}$ is contained in this kinematic range. The effect of finite reconstruction efficiency of $p$ and $\bar{p}$ has been shown to be negligible (17). The experimental values of $S \sigma$ and $\kappa \sigma^{2}$ are shown as a function of $\sqrt{s_{N N}}$.

The lattice calculations (16) were carried out using two flavors of staggered quarks in QCD. The lattice cutoff $1 / a \simeq 960-1000 \mathrm{MeV}$ and the bare quark mass were tuned to give a pion mass of about $230 \mathrm{MeV}$ (33). These computations were performed at $\mu_{B}=0$ and the Taylor series coefficients of $P / T^{4}$ were used to extrapolate $m_{1}$ and $m_{2}$ to the freeze-out conditions using appropriate order Padé approximants to resum the series expansions. Since lattice results are obtained in terms of $T / T_{c}$ and $\mu_{B} / T$ (see Eq. 1), their extrapolation to the freeze-out conditions required the input of $T_{c}$. The lattice values were obtained using $T_{c}=175 \mathrm{MeV}$, compatible with indirect determinations of $T_{c}(10-13)$.

On the upper scales of Fig. 3 we also show the $\mu_{B}$ and $T$ values at chemical freeze-out that correspond to the various $\sqrt{s_{N N}}$. For this we used the functional relationship between these values from the hadron resonance gas model using the yields of hadrons discussed in $(18,19)$. 


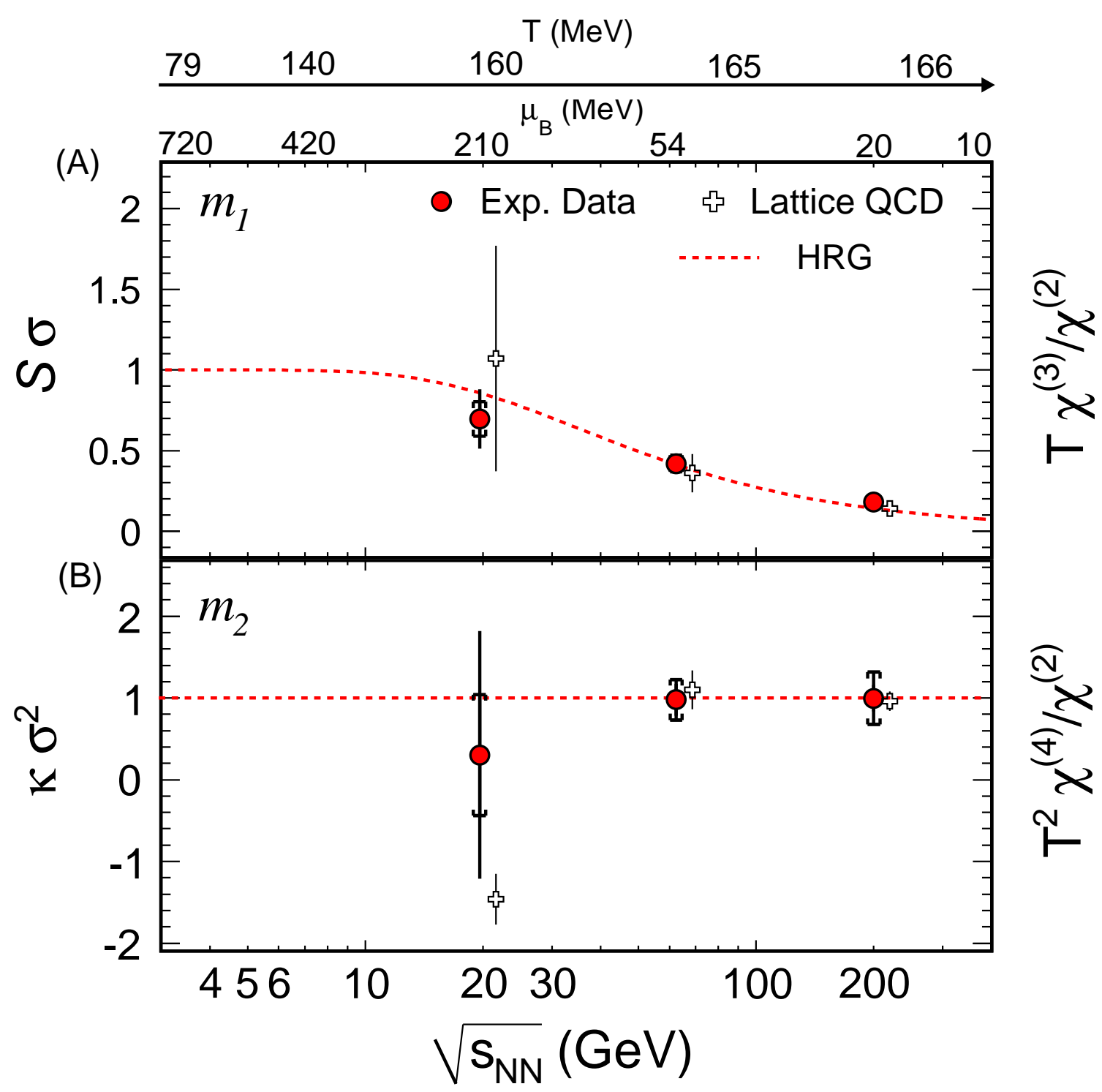

Figure 3: Comparison of lattice QCD and experimental data for $m_{1}(\mathrm{~A})$ and $m_{2}$ (B). Experimentally measured ratios of cumulants of net-proton distributions, $m_{1}=S \sigma$ and $m_{2}=\kappa \sigma^{2}$ are shown as a function of $\sqrt{s_{N N}}$ for impact parameter values of less than $3 \mathrm{fm}$ for $\mathrm{Au}+\mathrm{Au}$ collisions at RHIC (17). Also plotted on the top scale are the chemical freeze-out values of $\mu_{B}$ and $T$ corresponding to $\sqrt{s_{N N}}$ as obtained from a hadron resonance gas model, which assumes the system to be in chemical and thermal equilibrium at freeze-out $(18,19)$. The prediction of such a model for $m_{1}(34)$ is shown as the dashed red line. The lattice predictions for these quantities are drawn from a computation with lattice cutoff of $1 / a \simeq 960-1000 \mathrm{MeV}$ and converted to the dimensional scale of $T$ and $\mu$ using $T_{c}=175 \mathrm{MeV}$. 
The model predictions for $m_{1}(34)$ are also shown. The hadron resonance gas model predictions can be reproduced if baryon and anti-baryon numbers are independently Poisson distributed. Having established a connection between $\sqrt{s_{N N}}$ and $\left(T, \mu_{B}\right)$ we compare the experimental data on fluctuations to those predicted from lattice QCD. Excellent agreement is seen between lattice QCD predictions and experimental measurements for all three beam energies. This marks the first successful direct test of QCD against experimental data in the non-perturbative context of bulk hadronic matter. The agreement with the data is yet another non-trivial indication that the fireball produced in heavy-ion collisions is in thermal and chemical equilibrium at chemical freeze-out.

Setting the scale of bulk QCD: Lattice QCD results for $m_{1,2,3}$ are obtained for dimensionless arguments $T / T_{c}$ and $\mu_{B} / T$ as shown in Eq. 2. For a given value of $\sqrt{s_{N N}}$, the experimental observations are realized at the corresponding chemical freeze-out, characterized by $T$ and $\mu_{B}$. Thus a comparison of the two requires a choice of the scale, $T_{c}$. By varying this scale to obtain the best fit between the QCD predictions and experimental measurements, we determine $T_{c}$ for the first time through an observable connected to strongly interacting bulk matter. The results are, of course, subject to all the caveats expressed in the previous section. The observable that we choose for comparison is $m_{3}$. The lattice computation of this quantity has the smallest systematic uncertainties among the three explored here, and thus is the best quantity to use to constrain $T_{c}$.

Figure 4A shows the comparison of $m_{3}$ between experimental results from Au ion collisions and lattice QCD predictions. This is an extension of Fig. 3 which shows a comparison with $m_{1}$ and $m_{2}$. In this analysis, the results of $m_{1}, m_{2}$ and $m_{3}$ are consistent as required in Eq. 3, The new information here is that we show lattice predictions obtained with different values of $T_{c}$. The errors on the experimental data points are statistical (lines) and systematic (brackets) errors (17). The errors bars on the lattice predictions are statistical errors with a cutoff of 

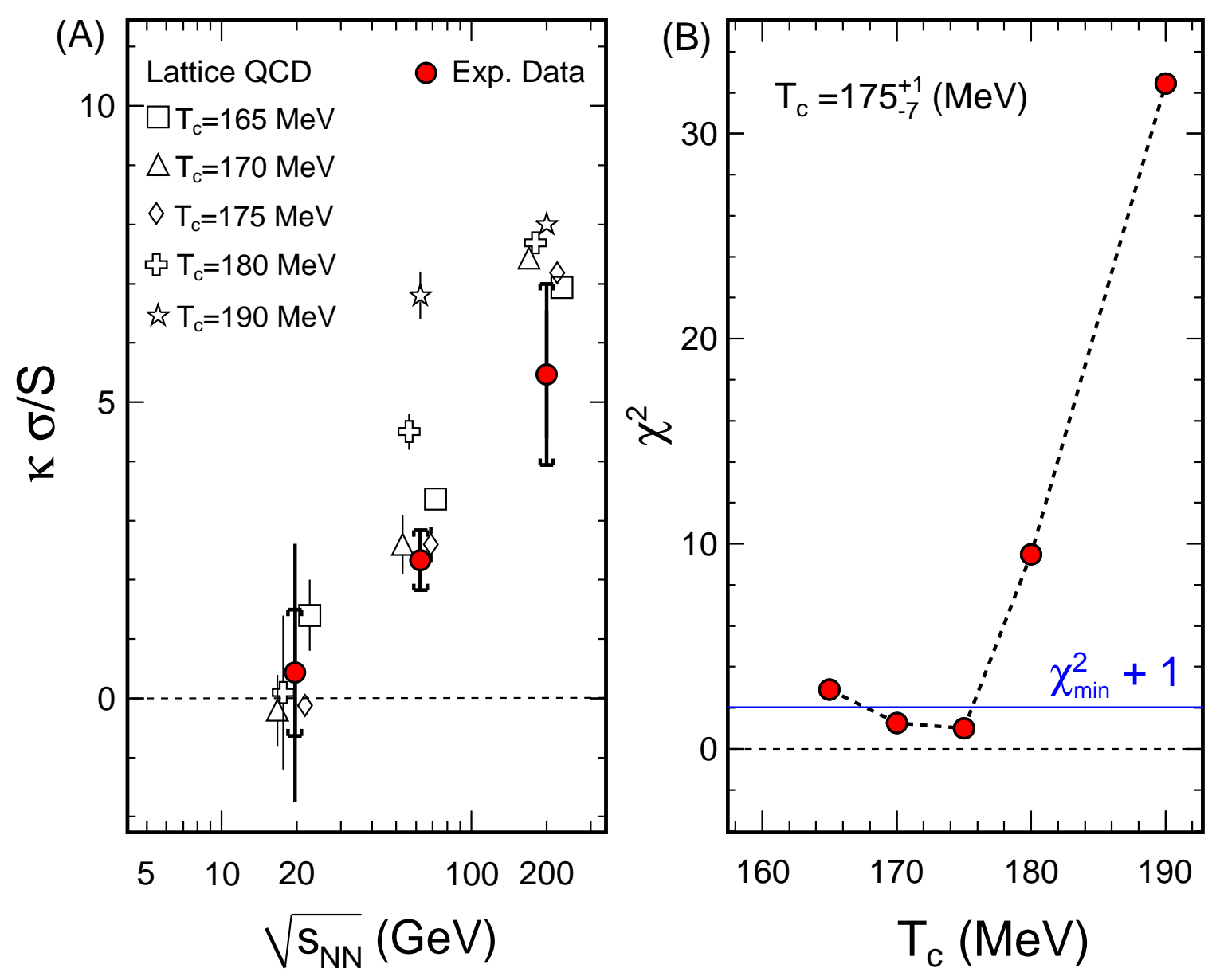

Figure 4: Comparison of $m_{3}$ from experiment and lattice predictions, and the extraction of $T_{c}$. (A): $\kappa \sigma / S$ of net-proton distribution measured in collisions of Au ions at varying $\sqrt{s_{N N}}$ and with an impact parameter of less than $3 \mathrm{fm}$. This is compared to lattice QCD predictions with cutoff $1 / a \simeq 960-1000 \mathrm{MeV}$ for the corresponding ratio of susceptibilities extrapolated to the freeze-out conditions using different values of $T_{c}$. The lattice results at each $\sqrt{s_{N N}}$ are slightly shifted for clarity in presentation. (B): The comparison of experimental data and lattice QCD predictions, shown through $\chi^{2}$ as a function of $T_{c}$ using the definition given in Eq. 4. This yields the estimate of $T_{c}$ and its errors as discussed in the text. 
$1 / a \simeq 960-1000 \mathrm{MeV}$. The lattice spacing effects and the effect of tuning the bare quark mass are the main sources of remaining uncertainties in the predictions. These are not parameterized as systematic uncertainties. However, it is known that their effect is small at the two highest values of $\sqrt{s_{N N}}(16)$.

In order to arrive at a quantitative estimate of the scale parameter $T_{c}$ we perform a standard statistical analysis. For each value of $T_{c}$ we compute,

$$
\chi^{2}\left(T_{c}\right)=\sum_{\sqrt{s_{N N}}} \frac{\left[m_{3}^{\operatorname{expt}}\left(\sqrt{s_{N N}}\right)-m_{3}^{\mathrm{QCD}}\left(\sqrt{s_{N N}}, T_{c}\right)\right]^{2}}{\text { Error }_{\text {expt }}^{2}+\text { Error }_{\mathrm{QCD}}^{2}}
$$

where the errors in the experimental and lattice QCD quantities are obtained as explained above. The lattice predictions are calculated for the grid of $T_{c}$ values (Fig. 4). The minimum of $\chi^{2}$, corresponding to the most probable value of the parameter being estimated, occurs at $T_{c}=$ $175 \mathrm{MeV}$. The standard errors on the parameter are the values of $T_{c}$ for which $\chi^{2}$ exceeds the minimum value by unity. It is clear from Fig.4(B) that this is bounded by +5 and $-10 \mathrm{MeV}$. A piece-wise linear interpolation between the grid points yields the more reliable error estimate, +1 and $-7 \mathrm{MeV}$. By comparing different interpolation schemes we find that the error estimate is stable. As a result we conclude that

$$
T_{c}=175_{-7}^{+1} \mathrm{MeV} .
$$

The error estimates include systematic and statistical errors on experimental data but only statistical errors on the lattice QCD computations.

The result in Eq. 5 is compatible with current indirect estimates of $T_{c}$ which come from setting the scale of thermal lattice QCD computations via hadronic observables. Furthermore, this gives a scale for temperatures which is compatible with the resonance gas model, as shown in Fig.3. As we discussed in the introduction, this closes a circle of inferences which shows that phenomena obtained in heavy-ion collisions are fully compatible with hadron phenomenology, and provides a first check in bulk hot and dense matter for the standard model of particle physics. 
Conclusions and Outlook: We have performed a direct comparison between experimental data from high energy heavy-ion collisions on net-proton number distributions and lattice QCD calculations of net-baryon number susceptibilities. The agreement between experimental data, lattice calculations and a hadron resonance gas model indicates that the system produced in heavy-ion collisions attained thermalization during its evolution. The comparison further enables us to set the scale for non-perturbative high temperature lattice QCD by determining the critical temperature for the QCD phase transition to be $175_{-7}^{+1} \mathrm{MeV}$.

This work reveals the rich possibilities that exist for a comparative study between theory and experiment of QCD thermodynamics and phase structure. In particular, the current work can be extended to the search for a critical point. In a thermal system, the correlation length $(\xi)$ diverges at the critical point. $\xi$ is related to various moments of the distributions of conserved quantities such as net-baryons, net-charge, and net-strangeness. Finite size and dynamical effects in heavy-ion collisions put constraints on the values of $\xi$ (35). The lattice calculations discussed here and several QCD-based models have shown that moments of net-baryon distributions are related to baryon number susceptibilities and that the ratio of cumulants $m_{2}=\kappa \sigma^{2}$, which is related to the ratio of fourth order to second order susceptibilities, shows a large deviation from unity near the critical point. Experimentally, $\kappa \sigma^{2}$ can be measured as a function of $\sqrt{s_{N N}}$ (or $T$ and $\mu_{B}$ ) in heavy-ion collisions. A non-monotonic variation of $\kappa \sigma^{2}$ as a function of $\sqrt{s_{N N}}$ would indicate that the system has evolved in the vicinity of the critical point and thus could be taken as evidence for the existence of a critical point in the QCD phase diagram.

\section{References}

1. F. Wilczek, Phys. Today 52N11, 11 (1999).

2. F. Wilczek, Phys. Today 53N1, 13 (2000). 
3. G. Sterman et al., Rev. Mod. Phys. 67, 157 (1995).

4. M. Peardon, AIP Conf. Proc., 1257, 19 (2010).

5. I. Arsene et al., (BRAHMS Collaboration), Nucl. Phys. A 757, 1 (2005).

6. B. B. Back et al., (PHOBOS Collaboration), Nucl. Phys. A 757, 28 (2005).

7. J. Adams et al., (STAR Collaboration), Nucl. Phys. A 757, 102 (2005).

8. K. Adcox et al., (PHENIX Collaboration), Nucl. Phys. A 757, 184 (2005).

9. B. I. Abelev et al., (STAR Collaboration), Phys. Rev. C 79, 034909 (2009).

10. S. Borsanyi et al., arXiv:1011.4330.

11. Y. Aoki et al., Phys. Lett. B 643, 46 (2006).

12. M. Cheng et al., Phys. Rev. D 74, 054507 (2006).

13. C. Schmidt (HotQCD Collaboration), arXiv:1012.2230.

14. F. R. Brown, N. H. Christ, Y. F. Deng, M. S. Gao, T. J. Woch, Phys. Rev. Lett. 61, 2058 (1988).

15. Y. Aoki, G. Endrodi, Z. Fodor, S. D. Katz, K. K. Szabo, Nature 443, 675 (2006).

16. R. V. Gavai, S. Gupta, Phys. Lett. B 696, 459 (2011).

17. M. M. Aggarwal et al., (STAR Collaboration), Phys. Rev. Lett. 105, 022302 (2010).

18. P. Braun-Munzinger, J. Wambach, Rev. Mod. Phys. 81, 1031 (2009).

19. J. Cleymans, K. Redlich, Phys. Rev. Lett. 81, 5284 (1998). 
20. M. A. Stephanov, K. Rajagopal, E. V. Shuryak, Phys. Rev. D 60, 114028 (1999).

21. R. D. Pisarski and F. Wilczek, Phys. Rev. D 20, 338 (1984).

22. S. Gupta, PoS, LATTICE2010, 007 (2010).

23. B. I. Abelev et al., (STAR Collaboration), Phys. Rev. C 81, 024911 (2010).

24. B. Mohanty, Nucl. Phys. A 830, 899c (2009).

25. Note that $\mu_{B}$ of bulk nuclear matter is quoted in the usual convention adopted in relativistic treatments, since anti-baryon production also needs to be accounted for. An alternative (non-relativistic) definitions which takes nucleon number to be fixed would give much smaller chemical potential. However, this does not correspond to the physics of baryon number fluctuations which we examine.

26. M. G. Alford, K. Rajagopal and F. Wilczek, Phys. Lett.B 422, 247 (1998).

27. R. V. Gavai and S. Gupta, Phys. Rev. D 68, 034506 (2003).

28. M. Asakawa et al., Phys. Rev. Lett. 85, 2072 (2000).

29. S. Jeon and V. Koch, Phys. Rev. Lett. 85, 2076 (2000).

30. Y. Hatta, M. A. Stephanov, Phys. Rev. Lett. 91, 102003 (2003).

31. S. Gupta, PoS, CPOD2009, 25 (2009).

32. The impact parameter is determined through a Glauber Monte Carlo procedure. The selected events correspond to the most central $0-5 \%$ and $0-10 \%$ events of the total hadronic cross section in $\mathrm{Au}+\mathrm{Au}$ collisions for $\sqrt{s_{N N}}=200,62.4$ and $19.6 \mathrm{GeV}$ respectively. 
33. The change in the radius of convergence in going from $m_{\pi} / m_{\rho}=0.33$ to 0.2 is likely to be between $10-15 \%$ (22). The corresponding effect on $m_{3}$ is about $2 \%$ or less at the two highest energies, and less than $20 \%$ at energy of $19.6 \mathrm{GeV}$.

34. F. Karsch, K. Redlich, Phys. Lett. B 695, 136 (2011).

35. B. Berdnikov et al., Phys. Rev. D 61, 105017 (2000).

36. We thank R. V. Gavai, F. Karsch, D. Keane, V. Koch, B. Mueller, K. Rajagopal, K. Redlich, H. Satz, M. Stephanov for enlightening discussions. We acknowledge the Indian Lattice Gauge Theory Initiative for computational support, DAE-BRNS through the project sanction No. 2010/21/15-BRNS/2026, US Department of Energy under Contract No. DE-AC0376SF00098 and the Chinese Ministry of Education.

This manuscript has been accepted for publication in Science. This version has not undergone final editing. Please refer to the complete version of record at http://www.sciencemag.org/. The manuscript may not be reproduced or used in any manner that does not fall within the fair use provisions of the Copyright Act without the prior, written permission of AAAS. 